\title{
ESPOROTRICOSIS FELINA: ASPECTOS CLÍNICOS E ZOONÓTICOS (1)
}

\author{
Carlos Eduardo LARSSON (2), Marcia de Ameida GONCALVES (2), Vera Cavalcanti ARAUJO (3), Maria Lucia Zaidan
} DAGLI (2), Benedito CORRFA (4) \& Celeste FAVA NETO (5)

\begin{abstract}
RESUMEN
La esporotricosis consiste en micosis profunda, de evolución subaguda o crónica decurrente de infección por el hongo dimorfico Sporothrix schenkii. Consiste en dermatopatia mucho frecuente en nuestro medio. resultante de la penetracion de abrojos e de aranazos por espinos de plantas. El S. schenkii ha sido descrito, en $\mathrm{S}$. Paulo, en perros. gatos, aseninos bovinos, equinos y ratones. Todavía, el carácter de antropozoonosis de la enfermedad poc as veces ha sido descrito tanto en la literatura internacional como en la nacional. existiendo apenas una citacion en lo Brasil. de probable transmisión gato hombre. Se discrebió, en lo presente relato, caso clínico de esporotricosis felina con transmision através de aranadura en propietario. tratador y médico veterinário. Un gato, sin raza definida, macho, con 3 años, mucho agresivo. con grave cuadro cutaneo (cefálico. torácico y de miembros torácicosi mani festado por lesiones ulceradas, exudativas de alopecia, deposición de crostas hemor. rágicas. agraviado por sintomas de lo complejo respiratório felino. donde por su temperamento agresivo habia areñado en corto espacio de tiempo 5 individuos. donde 3 de los individuos manifestaron sintomas de evolución y gravedad distintas. El diagnostico clínico presuntivo fue confirmado per lo examen histopatológico i H.E. PAS I de fragmentos de piel, linfonodos y amigdalas cogidos, "intra-vitam" y o "post morten". per lo aislamiento de lo agente, per la inoculación en testiculos de ratas adultas y posterior nuevo aislamiento.

Se confirmo la patologia por pruebas suerológicas (F.C., anticuerpos precipi. tantes) y prueba de intradermoreación (esporotriquinal en los pacientes acometidos.
\end{abstract}

UNITERMOS: Esporotricosis felina: Zoonosis: Gato.

\section{INTRODUCCION}

La esporotricosis consiste en micosis profun da causada por el hongo dimorfico Sporothrix schenkii, descrito por la primera vez por
SCHENCK $(1898)^{28}$, en los EUA, habiendo sido relatada en Brasil, por SPLENDORE \& LUTZ $(1907)^{30}$ en ratas y en hombres.

11) Apresentado no I Congresso Brasileiro de Zonnoses. Rin de Janeiro. Brasil. 1987 eno XXII Congresso Mundial de Veterinaria Montregl. Canada. 1987

(2) Departamento de Clínica Médica, Faculdade de Medicina Veterinária e Zootecnia da USP. Av. Prof Lúcio Martins Rodrigues. Trav. 4, 399. CEP 05508 Sào Paulo. SP. Brasil

13) Departamento de Estomatologia - Setor de Patologia Bucal - Faculdade de Odontologia da USP. Av. Prof Lucio Martins Rodiques Trav. 4. CEP 05508 Sào Paulo. SP. Brasil.

(4) Departamento de Microbiologia e Imunologia - Instituto de Ciencias Biomedicas da USP. Av Prof Lineu Prestes, 1374 CEP 05508 Sao Paulo. SP. Brasil.

(5) Laboratorio Bio Cicncias Lavoisier. Av. Angelica. 1832. CEP 01228 Sào Paulo. SP. Brasil 
LARSSON, C. E.; GONCALVES, M. de A.: ARAuJo. V. C.: DAGli, M. L. Z.: CORREA, B. \& FAVA NETO, C. Esporotricosis felina: aspectos clínicos e zoonoticos. Rev. Inst. Med. trop. S. Paulo, 31(5): 351 358, 1989

Según PUPO $(1920)^{20}$, SAMPAIO et al $(1954)^{26}$. ALMEIDA et al. $(1955)^{\prime}$ el diagnóstico de la infección fúngica en los diferentes cuadros clinicos de ella resultantes és asaz frecuente en pacientes atendidos en los Servicios de Derma tologia del Estado de Sāo Paulo (Brasilı. SAM PAIO et al. (1954) $)^{26}$ la colocan en segundo lugar en ocurrencia entre las micosis profundas suplantada únicamente por la paracoccidioidomicosis sudaméricana.

En la Medicina Veterinária, casos de esporo tricosis animal, en Brasil, han sido diagnostica dos en mulares (LEÄO et al., 1934"1): PIRATI NINGA, $1943^{19}$ : SALIBA et al, $1963^{25}$, en asini nos (MELLO, 1935 $5^{15}$ : MOREIRA et al., 1967'7). en perros (SOUZA, 1957"2: MIGLIANO et al.. $196364^{16}$ : LONDERO et al.. $1964^{12}$; FREITAS et al., $1956^{\circ}$ ), en chimpancés (SALIBA et al., 1968 ${ }^{24}$ ), en bovinos (MACEDO \& COSTA, 1978'1) y en gatos (FREITAS et al., 1965').

El S. schenckii comúnmente és identificado en el suelo, en vegetales, en el pelambre de ani males, en vetores mecanicos. como hormigas y pulgas, y mismo en embutidos como salsicha El acometimiento humano se hace por medio de heridas resultantes de la penetración de abro jos o de arañazos por espinos de plantas 1 AT TLEBERGER, $1983^{2}$.

Por la compulsación de in literatura algunos autores han sugerido la possibilidad de trans. mision de la enfermedad esporotricosica por medio del mordisco de ratas (FISCHMAN et al.. $1973^{\top}$ !, por arañazos, mordiscos o mismo por el contacto con felinos infectados (ALMEIDA et al., 1955': READ \& SPERLING, 1982"1: NUS BAUM et al., $1983^{18}$ ). Tal modalidad de trans misión és aún asi rechazada por otros pesquisa dores (BAXTER, 1975; RICHARD, 1975"2:) En Brasil, una única publicación describe la possi ble transmisión de la esporotricosis al hombre a partir de la arañadura del gato (ALMEIDA et al., 1955' , sin detenerse en mayores pormenores acerca de las manifestaciones clínicas en el ani. mal transmisor y en el individuo contaminado

En el presente relato se describe un caso de esporotricosis felina con el subsecuente envolvi miento de individuos que tuvieron contacto.

\section{RELATO DE CASO}

Se atendió junto al Servicio de Dermatolo gia del Departamento de Clinica Médica de la Facultad de Medicina Veterinária y Zootecnia de la Universidad de Sāo Paulo un espécimen felino, macho, SRD con tres años de vida provenido de Caraguata tuba (litoral norte de Sáo Pau lo), criado como animal querenciado, en contac to con otros dos gatos, entonces asintomáticos. Se relataba cuadro cutaneo de evolución de no venta dias anteriormente sometido tratamiento por curiosos y en Clínica Veterinária donde. por su temperamento agresivo habia arañado una mécica veterinária (M.C.A. veinte y siete años. soltera) y un tratador (J.P.S., veinte y cuatro años, solterol, hacian sesenta dias.

Los dueños observaron. inicialmente, un cuadro dermatológico caracterizado por lesio. nes cefalicas (región periorbitária) de alopecia, eritema, deposición de crostas hemorrágicas, acompanada por discreto prurito. Trahido a la Capital, fue entonces, medicado, bajo orienta ción veterinária, con cortisona, penicilina, vita mina A, ac pantotenico, tetraciclina, pasta de rgua, azufre y liquido de Dakin, visando la cura del supuesto cuadro de "sarna notoédrica infectada"

Después de los sesenta dias de medicación se averiguó la peora del cuadro lesional obser vandose lesiones diseminadas por toda la region cefálica, torácica dorsal, coccigea y a lo largo de los miembros torácicos. Habia alopecia y ul ceración, mayormente en la región craniana con pérdida de identidad de la fisura de los párpados y con exudación suerosanguinolenta.

Al examen clinico se constataba. además, disnea inspiratoria, taquinea iquarenta y ocho movimientos por minuto), normotermia $\left(38,5^{\prime \prime} \mathrm{C}\right.$ ), frequencia de pulso dentro de los parametros normales inoventa pulsaciones por minuto), in farto de ganglios linfáticos superficiales. secreción catarral naso ocular, bilateral y estornudos frecuentes. En la auscultación torácica se detec taron bullas normofoneticas eurritmicas. con propagación de los ruidos cardiacos para la re gión pulmonar, habiendo, todavia, discreta exa cerbación del murmurio vesicular y del laringotraqueal 
LARSSON. C. E.: GONÇALVES, M. de A.: ARAUJO, V. C.: DAGLI. M. L. Z.: CORREA, B. \& FAVA NETO. C. -

Después de coger la sangre (para hemogra ma y pesquisa de microfilárias) y fragmentos de piel para examen histológico, se prescribió tera. pia a base de cloranfenicol injetable, solución glicofisiológica a $5 \%$ por via parental, compres sas húmedas con solución de Burow y utilización de vapor húmedo bajo la forma de inalación en la tentativa de medicación del cuadro de complejo respiratório felino y de la dermatopatia.

Despues de diez dias, cuando del retorno se habia verificado flagrante mejora en el cuadro respiratório pero pocas alteraciones en el aspec to cutaneo, habiendo la ópcion por parte de los dueños por el sacrificio del animal. Después de la electrocución se cogieron, cuand ': de la necropsia fragmentos de piel, pulmón, ganglios linfáticos y amigdalas para examenes micológico y histopatológico.

En el transcurrir deste periodo contactandose con la médica veterinária y el tratador, se verificó que los mismos después de haberen sido arañados en las manos y antebrazos, manifes taran, casi veinte dias después del hecho cuadro febril recurriente, malestar, enfartamiento de ganglios linfáticos, cefalea, mialgia y proceso in flama tório en el sitio del arañazo.

El tratador llegó a ser internado en hospital paulistano con cuadro agudo de artrite en la articulación de la rodilla izquierda con un diagnós tico de sospecha de linforeticulose benigna de inoculación o enfermedad de la arañadura del gato (cat scratch fever). La médica veterinária presentó por meses dos heridas exudativas no cicatrizables de aspecto ulcerado en el antebrazo derecho quedandose. también bajo medicacion específica para enfermedad de la arañadura del gato.

Investigando junto a los duenos y contac tantes de la propriedad se verificó que tres personas habian sido arañadas en el transcurrir del tratamiento del animal. Una mujer (M.A., treinta años, soltera) y dos hombres (D.A., veinte y dos años, soltero y D.C., treinta y seis años, también soltero). La primera destos aranada en el pulgar de la mano derecha manifestó. cerca de siete dias después enfartamiento del linfonodo axilar derecho, mialgia en el brazo derecho, edema lo calizado y cefalea intermitente, habiendo sido examinada por vários médicos y sometida a inú meros examenes subsidiarios, estando desde en tonces sin diagnóstico y bajo medicación con sulfonamídicos/trimetropim y complejo vitami nico B. El contactante D.A., aranado en el miem bro torácico derecho manifesto "miosite" locali zada, habiendo sido medicado con sucesso con sulfonamidico trimetropim. Finalmente, el ter cer contactante. D.C., arañado en la mano iz quierda presentó discreto enfartamiento de lin fonodo axilar ipsilateral, mejorando sin cual quiera medicación

\section{EXAMES SUBSIDIÁRIOS}

\section{Hemograma y pesquisa de microfilárias}

Después de c og:r la sangre, por venopunc tura de la yugular del gato, usandose el EDTA como anticoagulante, se procedió al hemograma segun BIRGEL $(1982)^{\text {t }}$ y a la pesqusa de Dirofilaria immitis por las técnicas de la gota espesa y por el método de Knott modificado ( KNIGHT. $1983^{(0)}$.

\section{Examen histopatológico}

Se hizo la histopatologia de fragmentos de piel cogidos por biópsia por el punch de Keyes (4 y $6 \mathrm{~mm}$ ). sometiendo al procesamiento habi tual y a la coloración por las técnicas de HE, PAS. Cuando de la necrópsia, fragmientos de piel, iinfonodos, pulmón, amigdalas, lengua, ce rebro, higado, rinon, bazo, páncreas fueron, tam bién, sometidos a las técnicas histopatológicas citadas.

\section{Examen micológico}

Los examenes micológicos realizados a par tir de los fragmentos de piel cogidos por biópsia cutanea ou de "pool" de organos macerados, fue ron ejecutados en Agar-Sabouraud Dextrose acrecido de cloranfenicol ( $100 \mu \mathrm{g} \mathrm{ml}$ ) y en Myco biotic Agar Difco (Mycosel), incubandose a $25^{\circ} \mathrm{C}$ por treinta dias. La identificación de las colonias fue efectuada por medio del estudio macro e mi croscópico (RIEDDEL, $1950^{23}$ ) y por sembradura en Corn Meal Ágar (Difco).

El estudio de las características dimórficas de las colonias de $\mathbf{S}$. schencki fue realizada por medio del cultivo en ágar-infusión de cerebro corazón (BHI ágar-Difco) con incubación a $37^{\circ} \mathrm{C}$. 
LARSSON, C. E.; GONÇAlVES, M de A.: ARAUJO, V. C.; DAGli, M. L. Z.; CORREA. B. \& FAVA NETO. C. Esporotricosis felina: aspectos clínicos e zoonóticos. Rev. Inst. Med. trop. S. Paulo, 31(5): 351 . 358,1989

\section{Inoculación experimental}

Después de ejecutar la suspensión de la fase leveduriforme en água destilada estéril, se inoculó $0,5 \mathrm{ml}$ de suspensión espesa en testículo de ratas adultas.

\section{Prueba de intradermoreacción}

Se hicieron, además pruebas intradérmicas con histoplasmina, usandose el antigeno filtrado y con esporotriquina, usandose la suspensión de células leveduriforme muertas, preparada se. gún la técnica preconizada por CASTRO $(1960)^{5}$, inoculandose en diferentes momentos, intra dermicamente, $0,1 \mathrm{ml}$ de cada uno de los antigenos en el antebrazo de los pacientes sospechos, haciendose la lectura después de veinte y cuatro, cuarenta y ocho, setenta y dos horas, a contar de la inoculación.

\section{Reacciones suerologicas}

A partir del suero sanguíneo, cogido de 3 de los 5 pacientes expuestos al peligro de contágio (MCA, MA, DC), se hicieron las reacciones suerologicas de fijación de complemento y de anticuerpos precipirantes para $H$. capsulatum y $\mathbf{S}$. schenckii, según la técnica de Wadsworth et al modificada por FAVA NETO $(1955)^{6}$.

\section{RESULTADOS}

\section{Hemograma y pesquisa de microfilárias}

Por el eritrograma se ha verificado un cua dro de anemia regenerativa. En el leucograma se ha constatado leucocitosis por neutrofilia, eo sinofilia y monocitosis, y presencia de corpúsculos de Döhle en neutrófilos segmentados.

No se ha podido encontrar ninguna microfilária de $\mathbf{D}$. immitis por las técnicas utilizadas.

\section{Histopatologia}

En la piel, las mayores alteraciones fueron observadas al nivel dermico donde se visualizaba intensa infiltración de células mononucleares (macrófagos). En el interior de estas células al nivel citoplasmático se notaban corpúsculos ovalados, acidófilos, de aspecto leveduriforme, con pared colorandose intensamente por el PAS.
El aspecto morfométrico y tintorial ha permitido firmar el diagnóstico de parasitismo por Sporothrix schenckii.

En las amigdalas y ganglios linfáticos submandibulares y cervicales, se observaba en la región subcapsular $\mathrm{y}$ de los senos marginales grande cantidad de células mononucleares con citoplasma distendido por numerosas formacio nes semejantes a las descritas en la piel.

En la lengua se notaron descontinuidad epi telial y intenso infiltrado en las camadas submu cosa y muscular de células mononucleares, con citoplasma distendido y rellenado por los corpús culos leveduriformes, ya mencionados.

En los otros órganos no se detectaron altera ciones histológicas dignas de nota

\section{Examen Micológico}

Las colōnias de $\mathbf{S}$, schenckii isoladas, se pre sentaron, inicialmente, de color blanco, morfo metricamente pequeñas, sedosas y membranosas, haciendose, después negras y con micélio aéreo.

El microcultivo ha demonstrado hifas finas, septadas, con conídios piriformes o en forma de huevo, distribujendose a lo largo de las hifas, sesiles o pedunculados, disponiendose en forma do de "margarita".

En el BHI, a $37^{\circ} \mathrm{C}$ se observaron colonias de color crema, cerebriformes, y que microscopicamente tenian estruturas leveduriformes en la for. ma de naveta.

\section{Inoculación experimental}

Después de veinte dias de inoculación las ratas manifestaron cuadro de orquitis acentuada, obteniendose el aislamiento de $\mathbf{S}$. schenckii por el retrocultivo en ágar-Sabouraud-dextrosis, después de 5 dias $\left(25^{\circ} \mathrm{C}\right)$.

\section{Prueba de intradermorreacción}

La lectura de las in tradermorreacciones con histoplasmina se mostraron negativas en todos los pacientes. Ya aquellas hechas con la esporotriquina se mostraran positivas en 3 de los indivi 
LARSSON, C. E: GONCALVES. M. de A: ARAUJO, V. C.: DAGLi, M. L. Z.: CORREA, B. \& FAVA NETO. C. -

Esporotricosis felina: aspectos clínicos e zoonóticos. Rev. Inst. Med. trop. S. Paulo, 31(5): $351358,1989$.

duos sometidos a las pruebas (MCA, DC e JPS), con eritema y formación papuliforme midiendo en sus diametros, respectivamente, mas de $2 \mathrm{~cm}$ (M.C.A.) y con $1,5 \mathrm{~cm}$ (D.C. y J.P.S.).

\section{Reacciones sorológicas}

Los sueros de todos los individuos contactantes investigados estaban negativos frente al antigeno de $\mathbf{H}$. capsulatum. Mientras que, la reacción de fijación del complemento para $\mathbf{S}$. schenckii, hecha con el suero proveniente de la médica veterinária MCA, se ha mostrado positiva, hasta la dilución 23

\section{DISCUSIÓN}

La infección micotica por el S. schenckii en humanos és frecuente, mayormente con enfermedad ocupacional acometiendo individuos que por fuerza de la atividad profesional, como floristas y trabajadores del medio rural, vienem ame nudo contactando con esporos del hongo. Debido a la presencia de nodulos ulcerados o no y con envolvimiento linfoadenopático ascendien te (READ \& SPERLING, 1982 ${ }^{21}$ ), afeta, en la mayoria de las veces los miembros superiores y el rostro, siendo considerada rara en el tronco. Por veces, ha sido diagnosticada la forma extra cutânea, con localización osteo-articular podien do, esporadicamente, diseminarse hematogenicamente en el organismo (SAMPAIO et al., $1982^{27}$ ).

La esporotricosis felina, naturalmente adquirida, parece constituirse en una condición ra ra. Según READ \& SPERLING, $(1982)^{21}$, el artículo de los autores brasileños (FREITAS et al., $1965^{4}$, donde se describieron 8 gatos infectados por el S. schenckii, en periodo de dieciocho me ses en el Estado de São Paulo (Brasil) és la mayor colección de casos de esporotricosis felina dispuesta en la literatura mundial.

La disseminación de la infección hongica entre felinos parece estar bastante relacionada a sus hábitos, quales sean, de penetrar por entre nichos y friestas de material descartado, por el revolcarse frecuente en el suelo o en cascáras de arboles y por la atávica predilección de arañar otros felinos en el decorrer de juegos o de riñas. La esporotricosis felina ha sido reproduzida experimentalmente por meio de la supresión imu- nitaria con corticosteróides (MACDONALD et al., $1980^{13}$ ), hecho este que permite aventar la possibilidad del gato del presente relato, prove niente de zona endémica como la de Caragua tatuba, estar imunosuprimido por la infección viral (rinotraqueite o calicivirose) manifestada por los sintomas clasicos de la sindrome del com plejo respiratório felino.

El diagnóstico pudo ser estabelecido en lo que tañe al felino acometido, por los aspectos sintomáticos, lesionales y zoonóticos, asociados a los resultados revelados por el cuadro leucoci tário (leucocitosis debida a las neutrofilia, eosi nofilia y monocitosis).

Aunque no se tenga logrado éxito en coger el suero, face a las pésimas condiciones de hidra tación animal y del estado de sus venas yugula res, para la execución de las pruebas sorológicas. los resultados obtenidos en la histopatologia cu tánea, lengual y de los organos linfáticos, asocia dos al éxito en el aislamiento y demonstración del agente a partir de organos cogidos "intra vitan" y "post morten". permitieron la confirma ción diagnóstica. A estos ocurridos se soman el suceso obtenido en el reaislamiento del agente a partir del retrocultivo positivo obtenido a par tir del crescimiento del $\mathbf{S}$. schenckii de material proveniente de la orquitis, experimentalmente induzida.

Muy probablemente contribuyó para la gra ve evolución del cuadro clínico del gato acome tido por la esporotricosis, la imunosupresión acarreada por la superposición del complejo res piratório felino y por la indución iatrogénica, por la aplicacion de corticosteróides en el decurso morbido, tal como ya se ha demonstrado experi. mentalmente por la publicación de MACDO NALD et al. $(1980)^{13}$.

Se resalte que el diagnóstico diferencial con la modalidad cutánea de la dirofilariosis con otras micosis sistémicas (histoplasmosis, blasto micosis, criptococosis, feohifomicosis), con neoplasias de revestimiento cutáneo pudo ser esta blecido por medio de los resuitados histopato lógicos y de las culturas micológicas ejecutadas en diferentes momentos del curso de la enfermidad. 
LARSSON, C. E.; GONCALVES, M. de A.: ARAUJO, V. C.: DAGLi. M. L. Z.: CORREA, B. \& FAVA NETO. C. -

Esporotricosis felina: aspectos clínicos e zoonóticos. Rev. Inst. Med. trop. S. Paulo, 31(5): 351-358, 1989.

CUADRO 1

Cuadro sinoption de los casos felino y humano de la esporotricosis so pun los aspectos clinicos y laboratoriais

\begin{tabular}{|c|c|c|c|c|c|}
\hline Caso & Aspecto Clínico & $\begin{array}{l}\text { Aspecto } \\
\text { Micologico }\end{array}$ & $\begin{array}{l}\text { Intradermo } \\
\text { reacción }\end{array}$ & Sorologia: & $\begin{array}{l}\text { Inoculacion } \\
\text { Experimental }\end{array}$ \\
\hline $\begin{array}{l}\text { Felino, SRD } \\
\text { Marho. } 3 \text { anos }\end{array}$ & $\begin{array}{l}\text { Evolucion de } 90 \text { dias con le } \\
\text { siones cefalicas. toracica } \\
\text { dorsal. coccigea y de miem } \\
\text { bros toracicos con alopecia. } \\
\text { eritema. crosta hemorragi } \\
\text { ca. prurito discreto, ulceras } \\
\text { serosanguinolentas, pédi } \\
\text { da de identidad de la aber } \\
\text { tura palpcbral. Exámen Fi } \\
\text { sico: disnea inspiratoria. ta } \\
\text { quinea. normotermia. nor } \\
\text { moesfigmia. linfoadenome } \\
\text { galia. secrecion nasocular } \\
\text { catarral bilateral estornu } \\
\text { dos frequentes bulla nor } \\
\text { mofonetica se propagando } \\
\text { para los campos pulmona } \\
\text { res exacerbacion de los rui } \\
\text { dos respiratórios. }\end{array}$ & $\begin{array}{l}\text { Examen micologico } \\
\text { de la piel y de "pool" } \\
\text { de organos: } \\
\text { Sporothrix } \\
\text { schenckii }\end{array}$ & $\bullet$ & $\cdots$ & $\begin{array}{l}\text { Cobayas: orqui } \\
\text { te por Sporoth- } \\
\text { rix sehenckii }\end{array}$ \\
\hline $\begin{array}{l}\text { M.C.A. } 27 \text { anos } \\
\text { soltera. medica } \\
\text { veterinária }\end{array}$ & $\begin{array}{l}\text { Evolueión de cuadro por } \\
\text { aranadura en mano yen an } \\
\text { tebrazo derecho hace } 60 \\
\text { dias con lesión ulecrada no } \\
\text { cicatrizable exudativa, con } \\
\text { fiebre recurrente. linfoade } \\
\text { nopatia satélite ipsilateral. } \\
\text { cefalea. mialgia. inflama } \\
\text { cion local. }\end{array}$ & $\cdots$ & $\begin{array}{l}\text { Esporotriquina: } \\
\text { Positiva }(2 \mathrm{~cm}) \\
\text { Histoplasmina: } \\
\text { Negativa }\end{array}$ & $\begin{array}{l}\text { H. capsulatum: } \\
\text { Negativa } \\
\text { S. schenckii: } \\
123\end{array}$ & $\cdots$ \\
\hline $\begin{array}{l}\text { J.P.S. } 24 \text { anos } \\
\text { soltero. } \\
\text { tratador }\end{array}$ & $\begin{array}{l}\text { Evolucion del cuadro por } \\
\text { aranadura en las manos y } \\
\text { antebrazos hace } 60 \text { dias con } \\
\text { artralgia de la rodilla iz } \\
\text { quierda, claudicacion, mal } \\
\text { estar, cuadro febril recur } \\
\text { rente, cefalea, mialgiagene } \\
\text { ralizada. }\end{array}$ & $\cdots$ & $\begin{array}{l}\text { Esporotriquina: } \\
\text { Positiva } 11.5 \\
\text { cm) } \\
\text { Histoplasmina: } \\
\text { Negativa }\end{array}$ & $\begin{array}{l}\text { H. capsulatum: } \\
\text { Negativa } \\
\text { S. schenckii } \\
\text { Negativa }\end{array}$ & $\cdots$ \\
\hline $\begin{array}{l}\text { M.A. } 30 \text { anos } \\
\text { solteria. propie } \\
\text { taria }\end{array}$ & $\begin{array}{l}\text { Evolueion del cuadro por } \\
\text { aranadura en el pulgar dere } \\
\text { cho hace } 7 \text { dias, con linfoa } \\
\text { denopatia axilar ipsilateral. } \\
\text { mialgia del miembro supe } \\
\text { rior ipsilateral. inflamaeion } \\
\text { local y cefalea. }\end{array}$ & $\bullet$ & œ.• & $\begin{array}{l}\text { H. capsulatum: } \\
\text { Ncgativa } \\
\text { S. schenckii: } \\
\text { Negativa }\end{array}$ & •.• \\
\hline $\begin{array}{l}\text { D.C. } 36 \text { anos } \\
\text { soltero, propie } \\
\text { tario }\end{array}$ & $\begin{array}{l}\text { Evolueión del cuadro por } \\
\text { aranadura en el miembro } \\
\text { superior dereche con miosi } \\
\text { te localizada. }\end{array}$ & $\bullet$ & $\begin{array}{l}\text { Esporotriquina: } \\
\text { Positiva } 11.5 \\
\text { cm } \\
\text { Histoplasmina: } \\
\text { Negativa }\end{array}$ & $\begin{array}{l}\text { H. capsulatum: } \\
\text { Negativa } \\
\text { S.schenckii: } \\
\text { Negativa }\end{array}$ & œ.• \\
\hline $\begin{array}{l}\text { D.A. } 22 \text { anos } \\
\text { soltero propie } \\
\text { tario }\end{array}$ & $\begin{array}{l}\text { Evolucion del cuadro por } \\
\text { aranadura en la mano iz } \\
\text { quierda. con discreto infar } \\
\text { te del linfonodo axilar ipsi } \\
\text { lateral. }\end{array}$ & $\bullet$ & $\begin{array}{l}\text { Esporotriquina: } \\
\text { Negativa } \\
\text { Histoplasmina: } \\
\text { Negativa }\end{array}$ & $\begin{array}{l}\text { II. capsulum: } \\
\text { Negativa } \\
\text { S. schencki: } \\
\text { Negativa }\end{array}$ & $\cdots$ \\
\hline
\end{tabular}

* Agar Sabouraud dextrose con cloranfenicol, Mycobiotic Agar Difco, corn meal ágar, BHI Agar Difco.

Fijacion del complemento, anticuerpos precipitantes (FAVA NETO, 1955 ).

•. no realizado. 
LARSSON, C. E.; GONÇAlves, M. de A.: ARAujo, V. C.; DAGili, M. L. Z.; CORREA. B. \& FAva NETO. C. Esporotricosis felina: aspectos clinicos e zoonoticos. Rev. Inst. Med. trop. S. Paulo, 31(5): 351.358 .1989

Infelizmente no nos fue posible instituir una conduta terapéutica más adecuada a la esporo tricosis enfrente a la gravedad del cuadro y a la relutáncia de los propietários en tratarlo, debido al peligro contínuo de acometimiento humano, llevandolos entonces a optar por el sacrificio del gato.

Las formas clinicas observadas en los contactantes variaron entre la linfangítica (MA, DC, DA), la ulcerosa (MCA) y la extracutánea (JPS), siendo que en esta última el assentamiento al nível articular es considerada como de ocurrencia bastante rara (SAMPAIO et al., 1982 27 ). La evolución clínica en los pacientes acometidos osciló entre decurso benigno (MA, DC, DA) y crónico (MCA, JPS), La aplicación intradérmica de la esporotriquina probablemente contribuyó en los casos de evolución benigna, desde que SAMPAIO et al. (1982 $)^{27}$ creditan gran éxito a esta forma de vacinoterapia.

Los resultados obtenidos por las reacciones intradérmicas positivas, en 3 de los 5 indivíduos sintomáticos, expuestos al peligro de la infección fúngica $\mathrm{y}$ por el título sorológico $-23 \mathrm{de}$ la reacción de fijación del complemiento con antigeno de S. schenckii en la médica veterinária (MCA), nos permitieron apartar una sospecha de acometimiento por la "cat-scratch-fever" y presumir por un diagnóstico de esporotricosis.

La escasez de casos similares dispuestos en la literatura brasileña y estranjera nos llevaron a describir este surto esporotricótico de caracteres profesional y familiar, sirviendo para evidenciar el peligro en potencial, de la manipulación de animales, mayormente de la espécie felina, acometidos por lesiones úlcero-linfangiticas.

\section{SUMMARY}

\section{FELINE SPOROTRICHOSIS: CLINICAL AND ZOONOTIC ASPECTS}

The sporotrichosis is a deep mycosis, its course is subacute or chronic, and is caused by the Sporothrix schenckii. It's a very common dermatopathy, generally arising from thorn wounds, insects stings as well as from splinters. The S. schenckii has been described in Sāo Paulo, Brazil, in canines, felines, asinines, bovines, equines and murines. However, its antropozoo notic feature has seldom been mentioned in the international literature, and, in Brazil. there is only one report about a possible transmission cat-human being.

The current approach describes a clinical ca se of feline sporotrichosis transmitted by cat scratch to the owner, the career and the veteri narian. A very offensive three-year-old male mongrel cat showed severe cutaneous lesions in cephalic, thoracic regions and forelimbs. These lesions were ulcerations, exsudation, crusts, alo pecia worsened by the symptoms of the feline respiratory complex. This cat wounded, in a short time, 5 persons. Three of them had shown symptoms of distinct severity and development. The presumptive clinical diagnosis was corrobo rated by histopathology (HE, PAS) of skin. lym phonodes, and tonsils fragments obtained "in tra-vitam" and "post-morten". This was true by the isolation of the agent. Finally, this was con firmed as a result of serological (FC, precipita tion antibodies) and immunocutaneous (sporo trichina, histoplasmina) tests made in affected patients.

\section{REFERENCIAS BIBLIOGRAFICAS}

1. ALMEIDA. F : SAMPAIO, S. A. P.: LACAZ. C. S. \& FER
NANDES, J. C. - Dados estatisticos sobre a esporotricose
- analise de 344 casos. An bras. Derm. Sif., 1: 912,1955 .

2. ATTLEBERGER, M. H. - Subcutaneous and opportu nistic mycoses. In: KIRK, R. W., ed. - Current veterinary therapy, 7. ed. Philadelphia. Saunders. 1983. p. 11771178

3. BAXTER, D. L. - Superficial and deep mycotic infec tions. In: MOSCHELLA. S. L.: PILLSBURY, D. M. \& HUR LEY Jr., H. J. - Dermatology. Philadelphia. Saunders. 1975. p. 692695

4. BIRGEL. E. H. - Hematologia clínica veterinária. In: BIRGEL. E. H. \& BENESI, F. J., coord Patologia clinica veterinária. Sāo Paulo, Sociedade Paulista de Medicina Veterinária, 1982. p. 2-50.

5. CASTRO, R. M. - Prova da esporotriquina: contribuiçào para o seu estudo. Rev. Inst. A. Lutz (S. Paulo), 20; 582. 1960 .

6. FAVA NETO, C. - Estudos quantitativos sobre a fixaçào do complemento na blastomicose Sulamericana com anti genos polissacaridicos. Arq. Cirurg. clin. exp., 18: 197254. 1955

7. FISCHMAN, O.: ALCHORNE. M. M. A. \& PORTUGAL, M. A. S. C. - Human sporotrichosis following rat bite. Rev. Inst. Med. trop. S. Paulo, 15: $99102,1973$. 
LARSSON, C. E.: GONÇALVES, M. de A.; ARAUjO, V. C.: DAGLi. M. L. Z.: CORREA. B. \& FAVA NETO, C. Esporotricosis felina: aspectos clinicos e zoonoticos. Rev. Inst. Med. trop. S. Paulo, 31(5): 351358.1989.

8. FREITAS, D. C.; MIGLIANO. M. F. \& ZANI NETO, L - Esporotricose. Observação de caso espontàneo em gato doméstico. (F. catus, L.) Rev. Fac. Med.vet. Univ. S. Panlo, 5: $601-604.1956$.

9. FREITAS. D. C.: MORENO. G.: SALIBA. A. M.: BOT TINO, J. A. \& MOS. E. N. - Esporotricose em càes e gatos. Rev. Fac. Med. vet. Univ. S. Paulo, 7: 381387,1965

10. KNIGHT. D. H. - Heartworm disease. In: ETTINGER S. J. - T'extbook of veterinary internal medicine: disea ses of dogs and cats. 2. ed. Philadelphia, W. B. Saunders. 1983. p. 1097-1124

11. LEAO. A. E. de A.: SILVA Jr., O. \& PROENCA, M. -Sur un cas de sporotrichose à Sporotrichum beurmann observe par la première fois chez un mulet a Rio de Janei ro. C. R. Soc. Biol. (Paris), 116: $11571158,1934$.

12. LONDERO. A. T.: CASTRO. R. M. \& FISCHMAN. O. Two cases of sporotrichosis in dogs in Brazil. Sabouraudia, $3: 273274,1964$

13. MACDONALD. E.: EWERT, A. \& REITMEYER, J. C. Reappearance of Sporothrix schenckii lesions after admi nistration of Solu Medrol ${ }^{\mathrm{k}}$ to infected cats. Sabouraudia. 18: $295,300,1980$

14. MACEDO. M.M. \& COSTA. E. O.-Ocorrencia da esporo tricose -- infeccào em animais da especie bovina. Rev Fac. Med. vet. Zootec. Univ. S. Paulo, 15: $5968,1978$.

15. MELLO. A. - Um caso de esporotricose verucosa por Sporotrichum beurmann. Rev. Indust. anim., 2: 305314 1935

16. MIGLIANO. M. F. FREITAS. D C. \& MORENO. G. Esporotricose em cāes Rev. Fac. Med. vet. Univ. S. Paulo. i: 225235.196364

17. MOREIRA. E. C.: KASSAY. Y. \& BARBOSA. M - Espo rotricose em asinino no Estado de Minas Gerais. Arq. Ese. vet. Univ. Fed. M. Gerais, 19: 189 191, 1967.

18. NUSBAUM. B. P.: GULBAS. N. \& HORWITZ, S. N. -Sporotrichosis acquired from a cat. J. Amer. Acad. Derm., 8: $386 \cdot 391,1983$.

i9. PIRATININGA. S. N.-- Esporotricose em muar Rev. Fac Med. vet. Univ. S. Paulo, 2: $219222,1943$.
20. PUPO. J. A. - Sporotricose no Brasil. An paul. Med. Cirurg., 11:200, 207, 1920

21. READ, S. I. \& SPERLING, L. C. -- Feline sporotrichosis transmission to man. Arch. Derm.. 118: $429431,1982$.

22. RICHARD. J. L. - Sporotrichosis. In: HUBERT. W. T. MCCULLOCH. W. F. \& SCHNURENBERGER. P. R. Diseases transmitted from animals to man. 6 ed. Spring field, Charles C. Thomas, 1975. p. 488489 .

23. RIEDDEL. R. W...- Permancnt stained mycological pre paration obtained by slide culture. Mrcologia, 42: 265. 1950 .

24. SALIBA, A. M.; MATERA. E. A. \& MORENO. G. - Sporo trichosis in a chimpanzee. Mod. vet. Pract., 49: 74. 1968.

25. SALIBA, A. M.: SOERENSEN, B. \& MARCONDES VEI GA. J. S. -- Esporotricose em muar. Biologico (S. Paulo), 29: 209212,1963 .

26. SAMPAIO. S. A: LACAZ, C. da S. \& ALMEIDA. F. P - Aspectos clínicos da esporotricose em Sáo Paulo. Ana lise de 235 casos. Rev. Hosp. Clin. Fac. Med. S. Paulo. 9: 391402,1954

27. SAMPAIO, S. A. P.: CASTRO. R. M. \& RIVITTI. E. A - Dermatologia basica. 2, ed Sâo Paulo. Artes Medicas. 1982

28. SCHENCK, F. - On refractory sub cutaneous abcesses caused by fungus. possibly related to the Sporotrichia Johns Hopk. Hosp. Bull., 9: 286, 1898.

29. SOUZA. J. J. - Esporotricose em càes. In: CONGRESSO BRASILEIRO DE MEDICINA VETERINARIA. 7.. Recife 1957. Anais. p. 367371 .

30. SPLENDORE. A. \& LUTZ. A. - Sobre uma micose obser vada em homens e ratos. CContribuicao para o conheci mento das assim chamadas esporotricosesı. Rev. med. S. Paulo, 10: 433-450, 1907.

31. WADSWORTH. A. B. - Standard methods of the Division of Laboratories and Research of the New York Department of Health. Baltimore, Williams \& Wilkins. 1947

Recebido para publicaçà em 2021989 